hilar lymph nodes were involved. The histology of the main tumour showed oat cell carcinoma, while the removed subcarinal lymph node contained deposits of poorly differentiated squamous cell carcinoma. The patient $s$ progress was uneventful, though he was left hoarse following damage to the left recurrent laryngeal nerve. $\mathrm{He}$ did not receive radiotherapy or cytotoxic drugs. He has been carefully followed up for 14 years, and despite the bad prognosis initially given, he has remained quite fit, continuing at work, and clinically and radiologically he has shown no signs of recurrence of the lung cancer.

Mr. Abbey Smith lays down the criteria for acceptance of the existence of this phenomenon of cure following incomplete surgical resection of cancer as biop $y$ confirmation, record of incomplete surgery, and necropsy confirmation of absence of cancer through out the body. In the case described above, the first two criteria are fulfilled, but the third cannot be met since the patien remains alive after 14 years. It looks therefore as though the policy of Mr. Abbey Smith's unit of removal of the bulk of the tumour where complete removal is not possible, may at times-if but rarely-produce an unexpected successful result.am, etc.,

Redhill General Hospital,

Alex SAKUl

Surrey

\section{Acid-base Balance and Bleeding}

SIR,-The criticisms made by Mr. M. H Irving (29 May, p. 529) of our paper on acid-base balance in acute gastrointestinal bleeding (1 May, p. 242) prompt us to clarify certain points raised in his letter.

We reported that transfusion with stored bank blood, which is known to have a high lactate concentration ${ }^{1}$ and a low $\mathrm{pH},{ }^{2}$ resulted in a rise in arterial blood lactate concentration in seven patients who were not clinically shocked either before or after blood transfusion. Mr. Irving suggests that this was because the patients remained in a "state of compensated shock", and points out that "a normal arterial blood pressure is no guarantee that hypovolaemia has been corrected". These patients were all transfused to a normal central venous pressure ${ }^{3}$ prior to the second blood sample, with the exception of one patient who still had a low central venous pressure at this time. Blood volume measurements were carried out in five of these patients, including the patient with the low central venous pressure, using ${ }^{51} \mathrm{Cr}$ labelled red cells. All five patients had a blood volume well within the normal range, according to weight and sex, at the time of the second blood sample.

We also reported that patients who are clinically shocked may have a severe metabolic acidosis on admission. In one such patient we measured the blood $\mathrm{pH}$ again after transfusion of one litre of blood had reversed the state of clinical shock and raised the blood pressure to normal. There had been only a very slight increase in $\mathrm{pH}$ from $7 \cdot 25$ to $7 \cdot 26$, but a further increase to $7 \cdot 43$ occurred following infusion of $200 \mathrm{mEq}$ sodium bicarbonate. After this further blood transfusion was continued. Mr. Irving suggests that "the actual volume of the 200 $\mathrm{ml}$ of sodium bicarbonate solution was possibly as significant in correcting the acidosis as the buffer itself." It seems unlikely that such a small volume would produce such a marked effect when no significant effect had been obtained with one litre of blood. A be dangerous unless the patient's blood pH further point against this explanation is that the blood lactate concentration remained very high at $128 \mathrm{mg} / 100 \mathrm{ml}$ following the infusion of bicarbonate, as mentioned in our paper.

Mr. Irving complains that the authors "do not make it clear at what point they believe post-transfusion metabolic acidosis should be corrected. The implication, however, from the interval between their pre- and posttransfusion acid/base measurements is that it may be several hours after the cessation of blood transfusion." The measurements he is referring to here are those obtained in the patients who were not clinically shocked before or after blood transfusion, and we specifically point out that monitoring of acid-base balance "is probably unnecessary in patients who are not clinically shocked." We suggest, however, that it "is advisable in patients with acute gastrointestinal bleeding who are clinically shocked, especially if rapid blood transfusion is contemplated." We intended this to imply that these measurements should be carried out before or during the early stages of blood transfusion, while the patients are still clinically shocked. If a severe acidosis is detected, we would suggest correcting it immediately. We certainly did not imply that it should be corrected "several hours after the cessation of blood transfusion." Thus, in saying that his "main criticism of the paper is the suggestion that a persisten metabolic acidosis in shocked patients should be treated by the infusion of sodium bicarbonate" (our italics), Mr. Irving is misinterpreting our suggestion. We accept that eventually "the metabolic acidosis of hypovolaemic shock is self-correcting if adequate volume replacement is provided." Our point is that, in the acute situation of a patient in clinical shock with a severe lactic acidosis, the rapid transfusion of stored bank blood, with a high lactic acid concentration and a low pH, may is monitored.

In support of his opposition to the use of sodium bicarbonate, Mr. Irving quotes Schweizer and Howland's ${ }^{5}$ (1962) paper saying that the metabolic acidosis of shocked patients undergoing major surgery responds promptly to treatment of the hypovolaemia with acid bank blood. This paper has been superseded by their more recent publication on this subject, ${ }^{6}$ which is the one quoted in our paper. In this, they report an improved mortality rate with simultaneous infusion of alkali, and a similar improvement has been reported under more carefully controlled conditions in experimental animals with haemorragic shock. ${ }^{2}$

Mr. Irving further points out that sodium bicarbonate is "ineffective in treating the shock state." We did not at any stage suggest that it will correct the shock state, only the accompanying acidosis. We share his belief that correction of a low blood $\mathrm{pH}$ should not be allowed to divert attention from the need to correct hypovolaemia by blood transfusion.-We are, etc.

Guy's Hospital,

T. C. NORTHField

\section{Royal Infirmary}

B. J. KIRBY

Edinburgh

Hammersmith Hospital,

ANNE E. TATTERSFIELD

London $\mathrm{W} .12$

1 Gullbring, B., and Ström, G., Acta Medica Scandinavica, 1956, 155, 413

G. G., Manger, W. M., Mittelman, A. and Ultmann, J. E., Annals of the New York Academy of Sciences, 1961, 92, 596.
Northfield. T. C., and Smith, T., Lancet, 1970, 2. 584

Birkeland, S. J., Fournal of the Oslo City Hospitals, 1965, 15, 43.

Schweizer, O., and Howland, W. S., Surgery, Gynecology and Obstetrics, 1962, 114, 90

Howland, W. S., and Schweizer, O., in Clinical Anaesthesia, ed. L. R.

\section{Myocardial Infarction and the G.P.}

SIR,-Dr. L. Adamson (27 February, p. 505) warns against the use of prophylactic procainamide in early myocardial infarction in the absence of E.C.G. control.

In a recent report ${ }^{1}$ on experience in a coronary care unit an E.C.G. record was presented which illustrates one of the hazards to which he alludes (Fig.). It demonstrates sinus bradycardia, a run of ventricular premature beats, reversion to

sinus bradycardia, and then alteration to nodal bradycardia-all occurring in a period of less than one minute in a patient with fresh infarction. The rapid transition from tachy- to brady-arrhythmia is not uncommon in the early stages of infarction and is impossible to detect clinically.

In this case atropine could have precipitated ventricular fibrillation or tachycardia, and procainamide block-syndrome or asy-
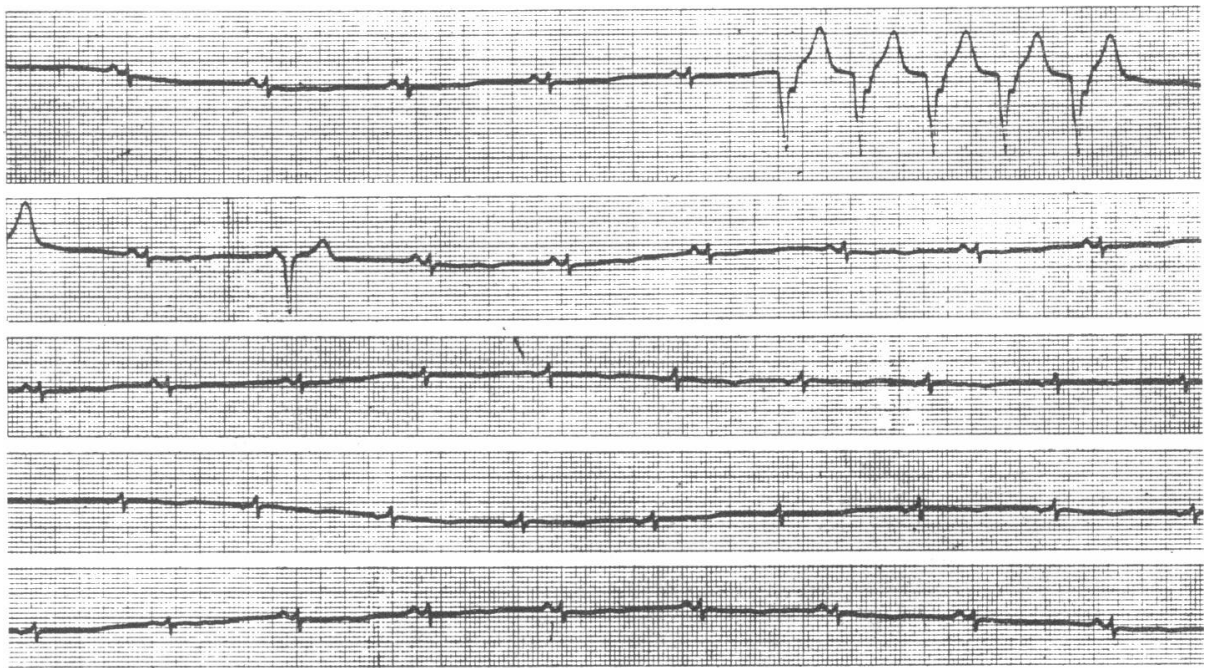